\title{
COMPROMETIMENTO DO PLEXO BRAQUIAL NA CIRURGIA CARDÍACA PARA REVASCULARIZAÇÃO DO MIOCÁRDIO POR ESTERNOTOMIA MEDIANA
}

\author{
AVALIAÇÃO CLÍNICA
}

MAURO ATRA*, ALBERTO ALAIN GABBAI**

\begin{abstract}
RESUMO - Para avaliar o plexo braquial na cirurgia cardíaca por esternotomia mediana para revascularização do miocárdio acompanhamos clinicamente 113 pacientes ( 87 homens e 26 mulheres) no pré-operatório e entre o $5^{\circ}$ e $8^{\circ}$ dia pós-operatório. Do total dos pacientes, em 65 foi utilizada a artéria torácica interna. Não foi realizado exame de eletroneuromiografia. Encontramos lesão do plexo braquial em três pacientes, sendo que apenas em um foi utilizada a artéria torácica interna. Acreditamos que fatores como postura do paciente, hipotermia, afastadores torácicos, uso da artéria torácica interna têm importância nestas lesões. Devemos ficar atentos a estes fatores para evitar ou minimizar as lesões.
\end{abstract}

PALAVRAS-CHAVE: plexo braquial, revascularização do miocárdio.

\section{The involvement of the brachial plexus in cardiac surgery with median sternotomy for the revascularization of the myocardium: clinical evaluation}

ABSTRACT -To evaluate the involvement of brachial plexus in cardiac surgery with median sternotomy for the revascularization of the myocardium 113 patients ( 87 men and 26 women) were clinically examined in the preoperative and between the fifth and eighth post-operative days. The internal thoracic artery was used in 65 of the 113 patients. The electroneuromyography was not effected in any of the patients. A lesion of the brachial plexus was found in three patients though the internal thoracic artery was used in only one patient.We believe that factors such as posture of the patient, hypothermia, thoracic braces and use of the internal thoracic artery are relevant in the lesions. Hence one must be attentive to all the factors mentioned above so as to avoid or minimize the lesions.

KEY WORDS: brachial plexus, myocardial revascularization.

O estudo das complicações neurológicas em cirurgia cardíaca tem despertado o interesse do neurologista. O número de cirurgias cardíacas e de outros procedimentos invasivos como cateterismo cardíaco vem aumentando. Existem muitos estudos mostrando o comprometimento do sistema nervoso central $(\mathrm{SNC})^{1-3}$ incluindo distúrbios psicológicos e comportamentais pós cirurgias cardíacas $^{1,4}$. Poucos autores, no entanto, estudaram o envolvimento do sistema nervoso periférico (SNP) após cirurgias cardíacas ${ }^{5,6}$. O comprometimento do plexo braquial após cirurgia cardíaca apresenta incidência que varia de $2 \%$ a $18 \%{ }^{1,7-9}$. A incidência é alta e o comprometimento do plexo é provavelmente causado pelo ato cirúrgico em si, quer pela posição do paciente durante o ato operatório, quer pela técnica operatória propriamente dita ${ }^{2,6}$ (Figura).

Estudo realizado na enfermaria de coronariopatia do Instituto Dante Pazzanese de Cardiologia: *Segundo Assistente de Neurologia da Santa Casa de São Paulo e Neurologista do Instituto Dante Pazzanese de Cardiologia;**Professor Titular de Neurologia da Universidade Federal de São Paulo - Escola Paulista de Medicina (UNIFESP-EPM). Aceite: 23-setembro-1999.

Dr Mauro Atra - Av Dr Altino Arantes 390/32 - 04042-002 São Paulo SP-Brasil. 


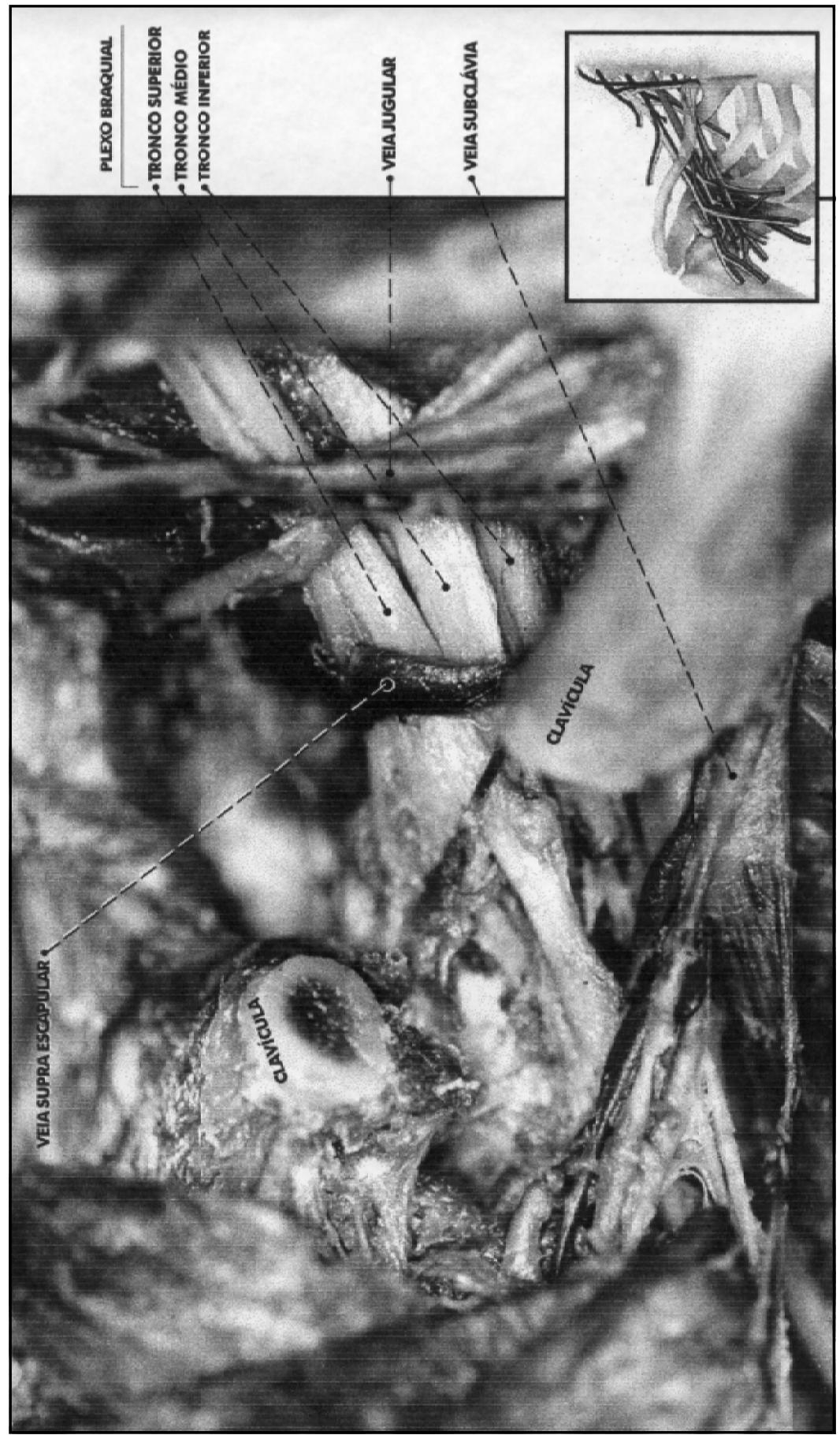

Figura. Região do plexo braquial após retirada de parte da clavícula, fotografada de cadáver da Disciplina de Anatomia Descritiva e Topográfica da UNIFESP-EPM. Permissão para fotografar gentilmente cedida pelo Prof. Dr. Ricardo L. Smith. 
$\mathrm{O}$ assunto do comprometimento do plexo braquial parece não causar muito interesse e muitas vezes passa para os cirurgiões, anestesistas e intensivistas como uma lesão central, um acidente vascular cerebral (AVC), principalmente quando há um só membro afetado. Sendo assim, podemos supor que o comprometimento do SNP possa ser maior do que o encontrado na literatura. Outros poucos anestesistas e grupos de cirurgia torácica também estudaram este problema na tentativa de prevenir ou minimizar as lesões ${ }^{10,11}$. Como neurologista do Instituto Dante Pazzanese de Cardiologia e observando lesões do plexo braquial, nervo frênico e mesmo plexo crural em pós-operatórios de cirurgia cardíaca, surgiu a idéia de estudar o envolvimento do plexo braquial pós-cirúrgico em nosso meio.

O objetivo deste estudo é avaliar clinicamente a frequência do comprometimento do plexo braquial, no pós-operatório, de pacientes submetidos a cirurgia cardíaca para revascularização do miocárdio com esternotomia mediana e tentar associar os achados com fatores operatórios como possíveis causas.

\section{MÉTODO}

Dentro de protocolo devidamente autorizado pela Comissão de Ética do Instituto Dante Pazzanese de Cardiologia, foram avaliados clinicamente pacientes internados na enfermaria de doenças coronarianas, para serem submetidos a revascularização do miocárdio de fevereiro a dezembro de 1997. Foram escolhidos para avaliação os pacientes que se internaram às segundas-feiras e apenas aqueles que eram encaminhados do ambulatório. Não participaram da avaliação os pacientes encaminhados do pronto-socorro ou aqueles que eram internados em caráter de urgência por qualquer motivo vindo de um procedimento do serviço de hemodinânica.

Avaliou-se um total de 130 pacientes, 97 homens e 33 mulheres. com idade média de 60 anos. Destes, 11 não foram operados porque após reavaliação com novo cateterismo cardíaco optou-se por outro procedimento como angioplastia ou angioplastia com "stent". Seis morreram por enfarte do miocárdio ou complicações cardíacas antes da cirurgia.

Com isso restaram 113 pacientes sendo 87 homens e 26 mulheres com idade mediana de 65 anos e média de 67,2 anos.

Os pacientes foram submetidos pelo primeiro autor a exame neurológico completo e exame neuromuscular específico para os membros superiores. Estes exames foram feitos na internação (pré-operatório) e entre o $5^{\circ} \mathrm{e} o$ $8^{\circ}$ dia pós-operatório. Não foram realizados exames eletrofisiológicos por motivos técnicos.

Os pacientes foram submetidos a esternotomia mediana para abordagem cardíaca. As bordas do esterno foram separadas e para mantê-las utilizou-se afastador de tórax tipo Anckeney colocado do terceiro espaço intercostal para baixo ${ }^{12}$. Os membros superiores durante a cirurgia permaneceram em paralelo em relação ao corpo. Todos os pacientes foram submetidos a monitorização cardíaca e da pressão arterial média, geralmente pela artéria radial. O catéter de acesso venoso profundo era subclávio direito em todos os pacientes estudados. Todos os pacientes foram colocados em circulação extra-corpórea por tempo médio de 60 minutos e neste período mantidos em hipotermia de $34^{\circ} \mathrm{C}$ em média ${ }^{12}$.

Nos 65 pacientes em que se utilizou a artéria torácica interna (anteriormente chamada artéria mamária interna) para revascularização, era elevada primeiramente a borda esternal esquerda para visualização da artéria e o hemitórax esquerdo era mantido tracionado usando-se afastador especial para este fim ${ }^{12}$. Após 30 minutos (tempo médio utilizado para a dissecção da referida artéria) era então trocado o afastador para um do tipo Anckeney para então prosseguir-se com a revascularização.

Os pacientes foram reavaliados entre o $5^{\circ}$ e o $8^{\circ}$ dia pós-operatório, pois já estavam extubados, fora da unidade de terapia intensiva (UTI), e com pouca ou sem dor no local da cirurgia. Com isso foram evitados fatores limitantes para o exame neurológico.

\section{RESULTADOS}

\section{Pré-operatório}

Nos 130 pacientes examinados encontramos 6 com sequelas de AVC caracterizadas por: paresia no hemicorpo esquerdo em quatro e no hemicorpo direito em dois. Os reflexos tendinosos apresentavam-se hiperativos do lado comprometido. Dois destes pacientes apresentavam espasticidade no membro superior, mais no punho e cotovelo com atrofia de musculatura interóssea. 
Em 16, dos 130 pacientes, encontramos hiporreflexia 1+ patelar bilateral e em 10 pacientes havia hiporreflexia $1+$ também dos aquileus.

Doze pacientes apresentavam alterações das sensibilidade dolorosa , táctil e térmica nas extremidades das mãos e pés, do tipo luva e bota.

\section{Pós-operatório}

Dos 113 pacientes operados, encontramos três $(2,6 \%)$ com comprometimento clínico do plexo braquial. Destes, dois eram homens e um mulher. Nos dois homens não foi utilizada a artéria torácica interna para a revascularização, na mulher, foi utilizada. Em um dos homens e na mulher encontramos comprometimento do tronco superior e médio do plexo braquial direito (paralisia tipo Erb-Duchenne), caracterizado como segue: Plegia dos músculos deltóide (parte anterior, média e posterior), biceps, supra-espinhal, infra-espinhal e braquiorradial. Hipoestesia dolorosa, táctil e térmica (quente e frio) na região do deltóide em sua parte anterior e média. Reflexos tendinosos bicipital 1+, tricipital 2+, braquiorradial 2+. Não observamos amiotrofia e não havia lesão trófica da pele mantendo a pilificação, cor e temperatura inalteradas. Unhas preservadas.

Além disso o paciente do sexo masculino teve tempo de perfusão de extracorpórea de 75 minutos, tempo de anoxia de 60 minutos e de hipotermia a $34^{\circ} \mathrm{C}$ de 70 minutos. A paciente do sexo feminino teve tempo de perfusão de extracorpórea de 80 minutos, tempo de anoxia de 60 e de hipotermia a $34^{\circ} \mathrm{C}$ de 70 minutos.

O outro paciente apresentava comprometimento do tronco inferior do plexo braquial (paralisia tipo Klumpke) esquerdo, caracterizado como segue: Paresia do músculo palmar longo, flexor superficial e profundo do carpo, adutor do polegar e abdutor do mínimo. Hipoestesia dolorosa,táctil e térmica (quente e frio) na face anterior da mão comprometendo região palmar e dorsal dos dedos mínimo e anelar e hipoestesia do antebraço parte interna, terço distal anterior e posterior. Reflexos tendinosos bicipital $2+$,tricipital $2+$, braquiorradial $2+$. Neste paciente também não encontramos alterações tróficas. Teve um tempo de perfusão de extracorpórea de 70 minutos, tempo de anoxia de 50 minutos e de hipotermia a $34^{\circ} \mathrm{C}$ de 60 minutos.

\section{DISCUSSÃO}

Cada vez menos aceita-se morbidade cirúrgica que se possa prevenir. O plexo braquial está vulnerável nas cirurgias cardíacas pelo posicionamento do paciente no leito cirúrgico, pelo tempo de permanência numa mesma posição, pela abertura do tórax e pela colocação dos afastadores torácicos ${ }^{2,6}$.

Encontramos em nossa casuística um porcentual de comprometimento baixo, 2,6\%, no limite inferior dos valores encontrados na literatura, que é de $2 \%$ a $18 \% \%^{2,5}$

A posição dos membros superiores era considerada causa importante de lesão de plexo braquial. Posicionava-se o paciente com flexão dos cotovelos em 90 graus e elevação de $30 \mathrm{~cm}$ em relação ao plano da mesa cirúrgica ${ }^{13}$. Posteriormente não mais elevavam-se os membros mantendo-se a flexão. Nestas duas posições provocava-se um estiramento do plexo braquial causado pela clavícula sobre o plexo. A cabeça do úmero também provocava o estiramento do plexo. Com isso e após observações dos pacientes optou-se por mudar o posicionamento do paciente mantendo-o em decúbito dorsal com os membros superiores em paralelo ao corpo. Este é o posicionamento usado nas cirurgias para revascularização do miocárdio e que foi adotado nos nossos pacientes ${ }^{6,11,13}$.

As diversas técnicas de abertura da caixa torácica pós esternotomia mediana são fatores potenciais importantes nas lesões do plexo braquial.

$\mathrm{O}$ afastador de tórax quando colocado para o procedimento e de acordo com o modelo (Favarolo, Anckeney) e modo de colocação pode vir a lesar o plexo. Quando o afastador é aberto 
acima do manúbrio esternal, a clavícula é empurrada para o interior do seu espaço no tórax, rodando sobre a primeira costela, comprimindo o plexo braquial. As raízes do plexo braquial têm sua saída em pontos próximos ao canal cervical e distalmente são presos à fáscia axilar. O afastador com o mecanismo citado estira as raízes do plexo braquial. Este também pode ser comprimido pela rotação da primeira costela, ou como alguns autores demonstram, a abertura excessiva do afastador pode fraturar, quebrar a primeira costela. É difícil constatar a fratura por radiologia simples convencional. Há necessidade de radiografia em projeção oblíqua da região cervical para visualizar ${ }^{8,14-17}$

Considerando-se o estiramento do plexo braquial como causa importante, haveria também diminuição na circulação sanguínea do plexo, às vezes formando pequenos hematomas por ruptura dos $\operatorname{vasos}^{8}$

A cateterização da veia jugular interna pela passagem de catéter de acesso venoso profundo, é causa importante de comprometimento do plexo braquial do mesmo lado da passagem. A lesão pode decorrer de agressão direta da agulha sobre os nervos ou pela formação de hematoma no local comprimindo o plexo ${ }^{1,10,12,18}$. Esta via de acesso não foi usada nos nossos pacientes. Utilizamos apenas a via subclávia direita. A hipotermia (entre $28^{\circ} \mathrm{C} \mathrm{e} 34^{\circ} \mathrm{C}$ ) que foi utilizada nos nossos pacientes e que é procedimento de rotina nas cirurgias cardíacas e sob circulação extracorpórea, pode ser um fator adicional nas lesões do plexo braquial ${ }^{3}$. O mecanismo pelo qual o plexo é comprometido não é conhecido; no entanto, parece ser importante o tempo de permanência em hipotermia,ou seja, um tempo mais prolongado apresenta maiores chances de produzir isquemia no plexo braquial ${ }^{3,5}$. Nos dois pacientes do nosso estudo que tiveram lesão do plexo braquial e em que não se utilizou a artéria torácica interna, alguns dos fatores acima citados podem ter contribuído. Podemos citar a colocação dos afastadores, tração sobre o plexo, hipotermia. Estes, isoladamente ou em conjunto, podem ter contribuído para a lesão.

Quando há indicação do uso da artéria torácica interna como proposta para revascularização, também encontramos outro fator de risco. Isto porque há necessidade da abertura da caixa torácica de forma assimétrica com um afastador de tórax específico para o procedimento. Afasta-se primeiro o hemitórax esquerdo até visualizar a junção costo-esternal. Este procedimento pode levar a fratura da articulação costo-esternal se for muito agressivo. Deve-se considerar que o tempo que se demora para dissecar a artéria torácica interna será o tempo que o hemitórax esquerdo permanecerá sob tração. Com isso podem ocorrer algumas complicações neurológicas ${ }^{9,13,19,20}$.

Tivemos em nosso estudo apenas um paciente com lesão do plexo braquial esquerdo, de um total de 65 pacientes que utilizaram a artéria torácica interna. Os cirurgiões que operaram nossos pacientes utilizaram um tipo especial de afastador torácico desenvolvido na divisão de Bioengenharia do Instituto Dante Pazzanese de Cardiologia. Ele mantém elevada a borda do hemitórax esquerdo sem levar a uma tração importante no plexo braquial e sem compressão da clavícula sobre o plexo. Com isso controla-se um fator importante de complicação neurológica, justificando a nossa pequena incidência de comprometimento do plexo.

Do exposto vemos que é importante conhecer e minimizar os fatores que tornam o plexo braquial mais exposto a lesões. Se observados em conjunto o benefício será para o paciente, com boa evolução pós-operatória e redução do tempo de hospitalização.

Os grupos de cirurgia cardíaca vêem as complicações neurológicas como uma morbidade importante a ser evitada em seus pacientes s, $^{1,5,21}$. Quando abordamos individualmente nossos cirurgiões cardíacos, eles também mostram-se cientes de tais complicações e de como evitá-las ou mesmo minimizá-las. Cabe ao neurologista conhecer melhor as complicações neurológicas da cirurgia cardíaca, no SNC e SNP, assim como avaliar os prováveis fatores de risco e manter uma interação com as equipes de cirurgia e clínica cardiológica ${ }^{3-5,22-24}$.

A evolução neurológica de uma lesão do plexo braquial geralmente é satisfatória ${ }^{25}$. Em nosso estudo dois pacientes tiveram evolução satisfatória. Foram os dois que não utilizaram a artéria torácica 
interna na revascularização do miocárdio. Encontram-se no momento bem e com boa movimentação do membro afetado (consulta telefônica após um ano). Isto é sugestivo de um bloqueio na condução nervosa, uma neuropraxia, por estiramento ou compressão nervosa ${ }^{25}$. Em um paciente o déficit permaneceu importante, com pouca melhora neurológica. Foi na paciente que usou a artéria torácica interna na revascularização. Ela provavelmente desenvolveu uma lesão axonal grave.

Considerando os fatores apresentados e os nossos achados, pudemos observar que mesmo sabendo dos riscos, com novos tipos de afastadores torácicos e mudanças de técnicas cirúrgicas, o plexo braquial continua suscetível a lesões . Devemos ficar atentos às morbidades neurológicas,neste caso ao SNP, mas também e reconhecidamente ao $\mathrm{SNC}^{4}$.

\section{REFERÊNCIAS}

1. Shaw PJ, Bates D, Cartlidge N, Heaviside D, Julian DG,Shaw DA. Early neurological complications of coronary artery bypass surgery. Br Med J 1985;291:1384-1387.

2. Furlan AJ, Sila CA, Chimowitz MI, Jones SC. Neurologic complications related to cardiac surgery. Neurol Clin 1992;10:145-166.

3. Brillman J. Central nervous system complications in coronary artery bypass graft surgery. Neurol Clin 1993;11:475-495.

4. Malheiros SMF, Brucki SMD, Bertolucci PHF et al. Neurological, outcome in patients subjected to cardiac surgery with and without cardiopulmonary bypass, (Abstract, 837P).Neurology 1993;43:A375.

5. Lederman RJ, Breuer AC, Hanson MR et al. Peripheral nervous system complications of coronary artery bypass graft surgery. Ann Neurol $1982 ; 12: 297-301$.

6. Jellish WS, Blakeman B,Warf P, Slogoff S. Hands-up positioning during asymmetric sternal retraction for internal mammary artery harvest: a possible method to reduce brachial plexus injury. Anesth Analg 1997;84:260-265.

7. Graham JG, Pye IF, McQueen IN. Brachial plexus injury after median sternotomy. J Neurol Neurosurg Psychiatry 1981; 44:621-625.

8. Stangl R, Altendorf-Hofmann A,Von Der Emde J. Brachial plexus lesions following median sternotomy in cardiac surgery. Thorac Cardiovasc Surg 1991; 39:360-364.

9. Vahl CF, Carl I, Müller-Vahl H, Struck E. Brachial plexus injury after cardiac surgery: the role of internal mammary artery preparation: a prospective study on 1000 consecutive patients. J Thorac Cardiovasc Surg 1991;102:724-729.

10. Hanson MR, Breuer AC, Furlan AF et al.. Mechanism and frequency of brachial plexus injury in open-heart surgery:a prospective analysis. Ann Thorac Surg 1983;36:675-679.

11. Jackson L, Keats AS. Mechanisms of brachial plexus palsy following anesthesia. Anesthesiology 1965;26:190-194.

12. Kirklin JW, Barrat-Boyes BG (eds). Cardiac surgery. 2Ed. New York: Churchil Livingstone, 1993:285-381.

13. Tomlinson DL, HirsCh IA, Kodali SV, Slogoff S. Protecting the plexus brachial during median sternotomy. J Thorac Cardiovasc Surg 1987;94:297-301.

14. Vander Salm TJ, Cereda JM, Cutler BS. Brachial plexus injury following median sternotomy. J Thorac Cardiovasc Surg 1980;80:447-452.

15. Vander Salm TJ, Cutler BS, Okike ON. Brachial plexus injury following median sternotomy. J Thorac Cardiovasc Surg 1982;83:914-917.

16. Baisden CE, Greenwald LV, Symbas PN. Occult rib fractures and brachial plexus injury following median sternotomy for open-heart operations. Ann Thorac Surg 1984;38:192-194.

17. Martin MA, Mari C, Miranda AF, Buron JA, Fernandez FE, Suarez R. Dolor por lesion del plexo braquial tras cirurgía de revascularizacion coronaria: a propósito de 3 casos. Rev Esp Anestesiol Reanim 1992; 39:180-182.

18. Rieke H, Benecke R, Devivie ER,Turner E, Crozier T, Kettler D. Brachial plexus lesions following cardiac surgery with median sternotomy and cannulation of the internal jugular vein. J Cardiothorac Anesth 1989; 33:286-289.

19. Roy RC, Stafford MA, Charlton JE. Nerve injury and musculoskeletal complaints after cardiac surgery: influence of internal mamary artery dissection and left arm position. Anesth Analg 1988; 67:277-279.

20. Hudson DA, Boome R, SANPERA I. Brachial plexus injury after median sternotomy. J Hand Surg [Am] 1993;18:282-284.

21. Mutarelli EG. Manifestações neurológicas e seus fatores preditivos na cirurgia para revascularização do miocárdio com CEC. Tese de Doutorado Faculdade de Medicina da Universidade de São Paulo. São Paulo, 1994.

22. Hickey C, Gugino LD, Aglio LS, Mark JB, Son LS, Maddi R. Intraoperative somatosensory evoked potential monitoring predicts peripheral nerve injury during cardiac surgery. Anesthesiology 1993;78:29-35.

23. Mickleborough LL, Walker PM,Takagi Y, Ohashi M, Ivanov J, Tamariz M. Risk factors for stroke in patients undergoing coronary artery bypass grafting. J Thorac Cardiovasc Surg $1996 ; 112: 1250-1259$.

24. Libman RB, Wirkowski E, Neystat M, Barr W,Gelb S, Graver M. Stroke associated with cardiac surgery. Determinants, timing, and stroke subtypes. Arch. Neurol 1997;54:83-87.

25. Ben-David B, Stahl S. Prognosis of intraoperative brachial plexus injury: a review of 22 cases. Br J Anaesth 1997;79:440-445. 